Nervenarzt 2009 [Suppl 1] · 80:12-13

DOI 10.1007/s00115-009-2778-1

(c) Springer Medizin Verlag 2009
P. Saemann ${ }^{1} \cdot$ M. Knop ${ }^{2} \cdot$ S. Lutz ${ }^{2}$ M. Czisch ${ }^{1} \cdot$ F. Weber ${ }^{2}$

${ }^{1}$ AG Neuroimaging, MPI für Psychiatrie, München

${ }^{2}$ AG Entzündliche Erkrankungen, MPI für Psychiatrie, München

\title{
Nachweis der Anreicherung von „Ultra Small Particles of Iron Oxide" in MS-Läsionen
}

\section{Ziel des Forschungsprojektes}

Die Multiple Sklerose (MS) zeigt eine Heterogenität hinsichtlich der Pathologie und der magnetresonanztomographischen (MRT)-Bildgebung zentralnervöser Läsionen, wobei histopathologisch definierte Subtypen dieser Läsionen durch nichtinvasive diagnostische Mittel bisher nicht unterschieden werden können. Erste Untersuchungen weisen darauf hin, dass diese MS-Subtypen möglicherweise mit dem Ansprechen auf unterschiedliche Therapien korrelieren. Da routinemäßige Hirnbiopsien bei MS-Patienten mit typischen Symptomen nicht gerechtfertigt sind, spielen nichtinvasive MRTTechniken zur Gewebecharakterisierung, wie z. B. DTI (Diffusion Tensor Imaging), ${ }^{1} \mathrm{H}-\mathrm{MR}$-Spektroskopie und spezifischere Kontrastmittel, eine zunehmende Rolle. Ultra Small Particles of Iron Oxide (USPIO) werden von Makrophagen phagozytiert und reichern sich in Läsionen des Zentralnervensystems bei Tieren mit experimenteller allergischer Enzephalomyelitis (EAE) - dem gebräuchlichsten Tiermodell der MS - an. In der EAE stellen USPIO die Einwanderung von Makrophagen in die Läsionen dar, während Gadolinium (Gd) die Störung der Blut-HirnSchranke anzeigt. Erste Studien bei MSPatienten zeigten, dass durch USPIO Makrophagen/Monozyten in vivo im Bereich von MS-Läsionen angefärbt werden können und dass die USPIO-Aufnahme nicht an eine Blut-Hirn-Schranken-Störung gekoppelt ist. Bisher liegen jedoch keine Un- tersuchungen zur USPIO-Kontrastierung bei Patienten ohne klinische Schubaktivität oder systematische, prospektive Untersuchungen einschließlich klinisch-radiologischer Korrelationen vor.

Haupthypothese der Studie ist, dass subklinische und nicht mit Gd erfasste Entzündungsaktivität durch USPIO quantifiziert werden kann. Ein besonderer Schwerpunkt liegt auf der Untersuchung der USPIO-Charakteristika der weißen Substanz in Abhängigkeit von der T1-Intensität, die im Extremfall („black holes") eine schwere stattgehabte Matrixschädigung anzeigt.

\section{Methoden}

Neunzig MS-Patienten (McDonald-Kriterien, davon $50 \%$ behandelte und $50 \%$ unbehandelte Patienten) und 15 Patienten mit klinisch isoliertem Syndrom (CIS) erhalten eine einmalige Gabe von USPIO ( $40 \mu \mathrm{mol} / \mathrm{kg}$ Körpergewicht, intravenös) sowie eine 6-monatliche klinische Untersuchung (Expanded Disability Status Score und Multiple Sclerosis Functional Composite) und MRT-Nachbeobachtung über mindestens ein Jahr. Das MRT-Protokoll umfasst vor Gd-Gabe (Tag 1): hochaufgelöster T1-gewichteter 3D-Datensatz (3DDS), axiale FLAIR (Fluid Attenuated Inversion Recovery)-, $\mathrm{T}_{1}-, \mathrm{T}_{2}$ - und $\mathrm{T}_{2}{ }^{*}$ Sequenz, diffusionsgewichtete Bilder. Nach Gd-Gabe (Tag 1): T1-3DDS, axiale $\mathrm{T}_{2}{ }^{*}$ - und T2-Sequenz. $24 \mathrm{~h}$ nach USPIOGabe (Tag 2): $\mathrm{T}_{1-3} \mathrm{DDS}$; axiale $\mathrm{T}_{2}{ }^{*}$ - und T2-Sequenz. An einer Patientensubgrup- 


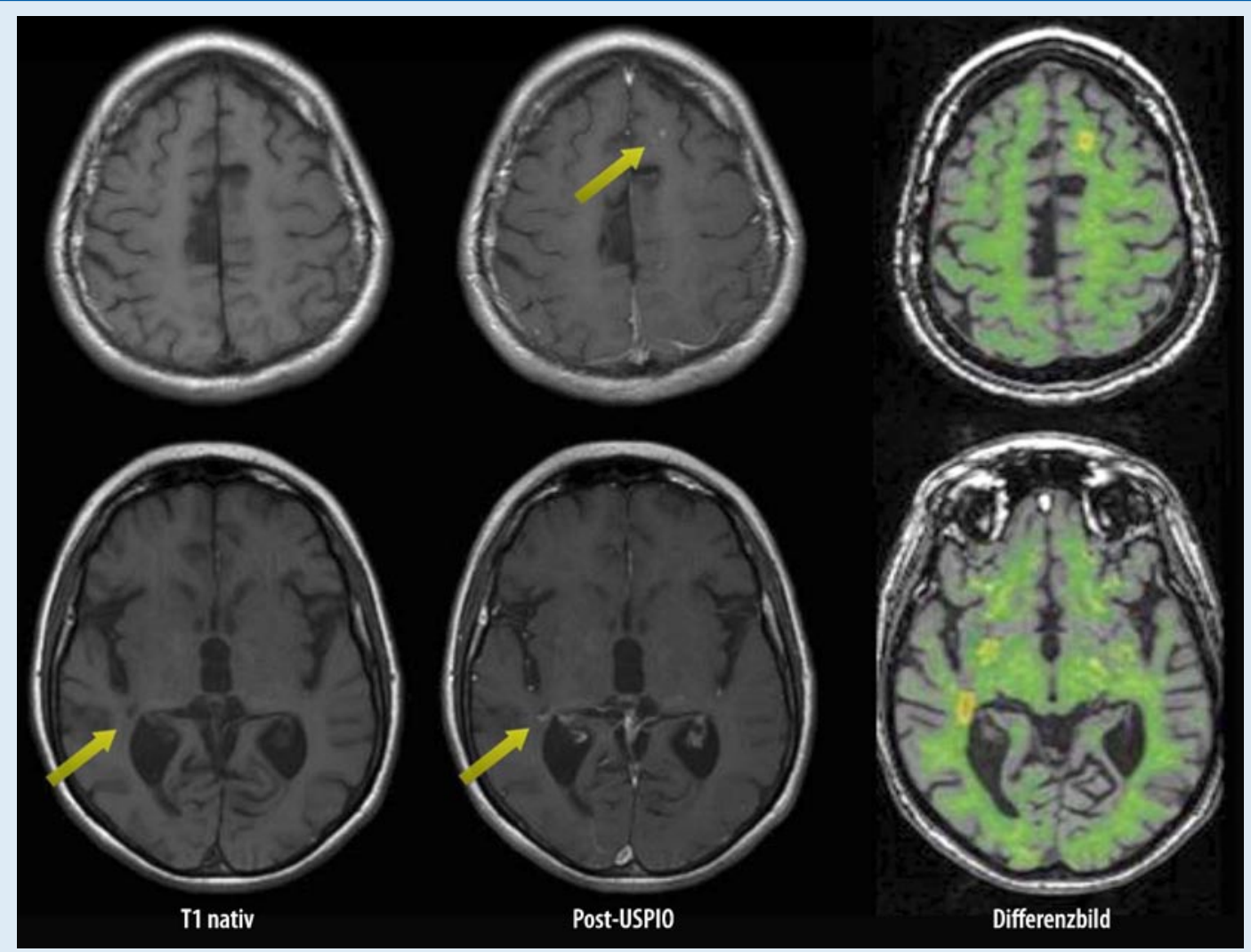

Abb. 1 A T1-Signalanhebungen durch USPIO: Vergleich der visuellen Analyse mit Differenzkarten. Links: Darstellung von nativen, axialen T1-gewichtete Bilder eines Studienpatienten mit Zeichen der inneren Hirnatrophie und einer T1-hypointensen Läsion in Nachbarschaft zum linken Seitenventrikelunterhorn (Pfeil). Mitte: $24 \mathrm{~h}$ nach USPIO-Gabe leichte fokale Signalanhebungen randständig im Bereich der (s. oben) T1-Läsion und zusätzlich Nachweis einer zweiten, kleinen fokalen Signalanhebung im linksfrontalen Marklager. Rechts: Differenzkarte zwischen Post-USPIO-Bild und T1-nativ-Bild nach Inhomogenitätskorrektur und Koregistrierung. Die Differenzwerte wurden auf den Mittelwert 0 und einen konstanten Varianzwert normiert; die Farbkodierung zeigt in grün Differenzwerte um Null, in gelb und rot höhere (positive) Differenzwerte. Die Differenzmethode ist geeignet, um USPIO-bedingte Signalanhebungen sensitiver zu erfassen. Weiterführende quantitative Auswertungen werden daher auf Differenzkarten von T1-gewichteten Bildern und absolut quantifizierten T1-Karten (T1-Relaxometrie) durchgeführt

pe wird eine absolute T1-Quantifizierung zur Methodenvalidierung vorgenommen. Die Auswertung wird sich auf den T1oder $\mathrm{T}_{2}{ }^{*}$-Intensitätsunterschied vor/nach USPIO-Gabe fokussieren. Dieser Intensitätsunterschied wird in Abhängigkeit von der Floridität gemessen an der Gd-Aufnahme und der nativen T2/FLAIR- sowie T1-Intensität untersucht. Zusätzlich werden voxelweise Differenzkarten erstellt, um den USPIO-Effekt anatomisch exakter lokalisieren zu können.

\section{Ergebnisse}

Zwanzig MS-Patienten wurden untersucht. Eine Patientin erlitt im zeitlichen Zusammenhang mit der USPIO-Gabe einen Harnverhalt, der $24 \mathrm{~h}$ später spontan remittierte. Sonstige unerwünschte Er- eignisse wurden nicht beobachtet. Eine visuell erfassbare USPIO-Anreicherung war bisher in einem Fall eindeutig, der eine gleichzeitige Gd-Anreicherung zeigt. Die Differenzkarten (• Abb. 1) erwiesen sich als sensitiver als die visuelle Analyse in Bezug auf die Darstellung der räumlichen Ausdehnung der USPIO-Läsionen. Qualitativ zeigten die Läsionen eine periphere Akzentuierung.

\section{Schlussfolgerung}

Zusammenfassend scheinen starke, visuell greifbare USPIO-Kontrastierung bei MR-tomographisch nicht-florider MS eher selten zu sein. Automatische quantitative Analysemethoden, wie die voxelweise Subtraktion nach Koregistrierung, können hierbei die Sensitivität erhöhen.

\section{Korrespondenzadresse}

\section{Prof. Dr. med. F. Weber}

AG Entzündliche Erkrankungen,

MPI für Psychiatrie

Kraepelinstraße 10, 80804 München

fweber@mpipsykl.mpg.de

Interessenkonflikt. Der korrespondierende Autor gibt an, dass über die Interessenkonflikte „Vertragshonorar",,"Reisekostenerstattung " und „,Forschungsunterstützung durch die Bayer Vital GmbH/Bayer Schering Pharma AG kein weiterer Interessenkonflikt besteht. 